\title{
HUMAN-IN-THE-LOOP DESIGN WITH MACHINE LEARNING
}

\author{
Wang, Pan (1); Peng, Danlin (1); Li, Ling (2); Chen, Liuqing (1); Wu, Chao (3); Wang, Xiaoyi (3); \\ Childs, Peter (1); Guo, Yike (1) \\ 1: Imperial College London, United Kingdom; 2: University of Kent, United Kingdom; 3: Zhejiang \\ University, China
}

\begin{abstract}
Deep learning methods have been applied to randomly generate images, such as in fashion, furniture design. To date, consideration of human aspects which play a vital role in a design process has not been given significant attention in deep learning approaches. In this paper, results are reported from a humanin-the-loop design method where brain EEG signals are used to capture preferable design features. In the framework developed, an encoder extracting EEG features from raw signals recorded from subjects when viewing images from ImageNet are learned. Secondly, a GAN model is trained conditioned on the encoded EEG features to generate design images. Thirdly, the trained model is used to generate design images from a person's EEG measured brain activity in the cognitive process of thinking about a design. To verify the proposed method, a case study is presented following the proposed approach. The results indicate that the method can generate preferred designs styles guided by the preference related brain signals. In addition, this method could also help improve communication between designers and clients where clients might not be able to express design requests clearly.
\end{abstract}

Keywords: Machine learning, Artificial intelligence, Design cognition, Computational design methods

Contact:

Wang, Pan

Imperial College London

Dyson school of Design Engineering

United Kingdom

pan.wang15@imperial.ac.uk

Cite this article: Wang, P., Peng, D., Li, L., Chen, L., Wu, C., Wang, X., Childs, P., Guo, Y. (2019) 'Human-in-the-Loop Design with Machine Learning', in Proceedings of the 22nd International Conference on Engineering Design (ICED19), Delft, The Netherlands, 5-8 August 2019. DOI:10.1017/dsi.2019.264 


\section{INTRODUCTION}

Automatically generating a design with preferences has been an on-going challenge in the design domain. Many deep learning methods have been proposed to generate designs. For example, image style transfer (Gatys et al., 2016)(Efros \& Freeman 2001)(Dosovitskiy \& Brox 2016)(Isola et al., 2017a) composes an image with original content and style features. Generative bionics design (Yu et al., 2018) employs an adversarial learning approach to generate images containing both features from the design target and biological source. However, these AI image generation methods do not consider human aspects, and the randomly generated results have no human cognition input. Consideration of human aspects in the design process is vital in design (Vicente 2013; Carroll 2002; Cooley 2000). A person's preference for a design can be intuitive, and sometimes an individual may not precisely know what their real preferences are. Therefore, being able to capture human preference in the image generation process to produce an image corresponding to a preference may lead to a significant improvement in AI-aided generative design. Recent advancements in neuroscience, especially brain decoding (Tirupattur et al., 2018; Shen et al., 2017; Palazzo et al., 2017) based on deep learning methods show potential for reconstructing a seen or imagined image from brain activities such as by using electroencephalogram (EEG), functional magnetic resonance imaging (FMRI) and Near-Infrared Spectroscopy (NIRS). This has provided impetus to explore a human-in-the-loop AI design method through deep learning as presented in this paper.

In this study, an attempt has been made to add human cognition into a deep learning based design process to generate design images which account for a person's preference. Human cognition involves many factors. In order to limit the scope of attention here, only human preference for potential styles has been explored. A human-in-the-loop AI design method is proposed, with a brain signal conditioned generative neural networks (GAN) (Goodfellow et al., 2014) framework for design, enabling cognitive visual-related styles to be reconstructed. Figure 1 illustrates a diagram of the proposed process. To implement this framework, an image presentation experiment is used to explore the relation between the brain signal and images presented by training an encoder to extract the features from raw EEG data when viewing the image. Secondly, a generator is trained using a GAN framework conditioned by the encoded brain signal features to reconstruct the presented image. Such a trained model is then used to reconstruct real preferred design images in a preference imagery experiment. Given the brain signal related to the imagination of a preferred design as input, the trained model could be used to generate images that probably contain the preferences.

Both qualitative and quantitative experiments were conducted for a case study and it was shown that the proposed human-in-the-loop AI design method could generate some design images people preferred. The experiment successfully demonstrated that desired design images can be generated using the brain activity which was recorded while subjects are imagining a product they prefer. The human-in-the-loop design approach could be embedded directly into other design processes with the understanding of design cognition incorporated. For example, by using this approach in fashion and product design, one could explore the cognition of possible preference on materials, patterns and shapes. Such learned design cognition could contribute to better design choices. This approach can also provide a new way for personalised design, for example a personalised gift design with customisation for the recipient.

The main contributions of this paper can be summarised as follows.

1) A human-in-the-loop AI design method to generate designs taking into account the subject's preference by employing EEG measured brain activity.

2) A new framework for communicating the cognitive understanding of customer requirements, enabling, for example, designers to have a visual understanding of what their clients want or their ideas through pictures not words. 


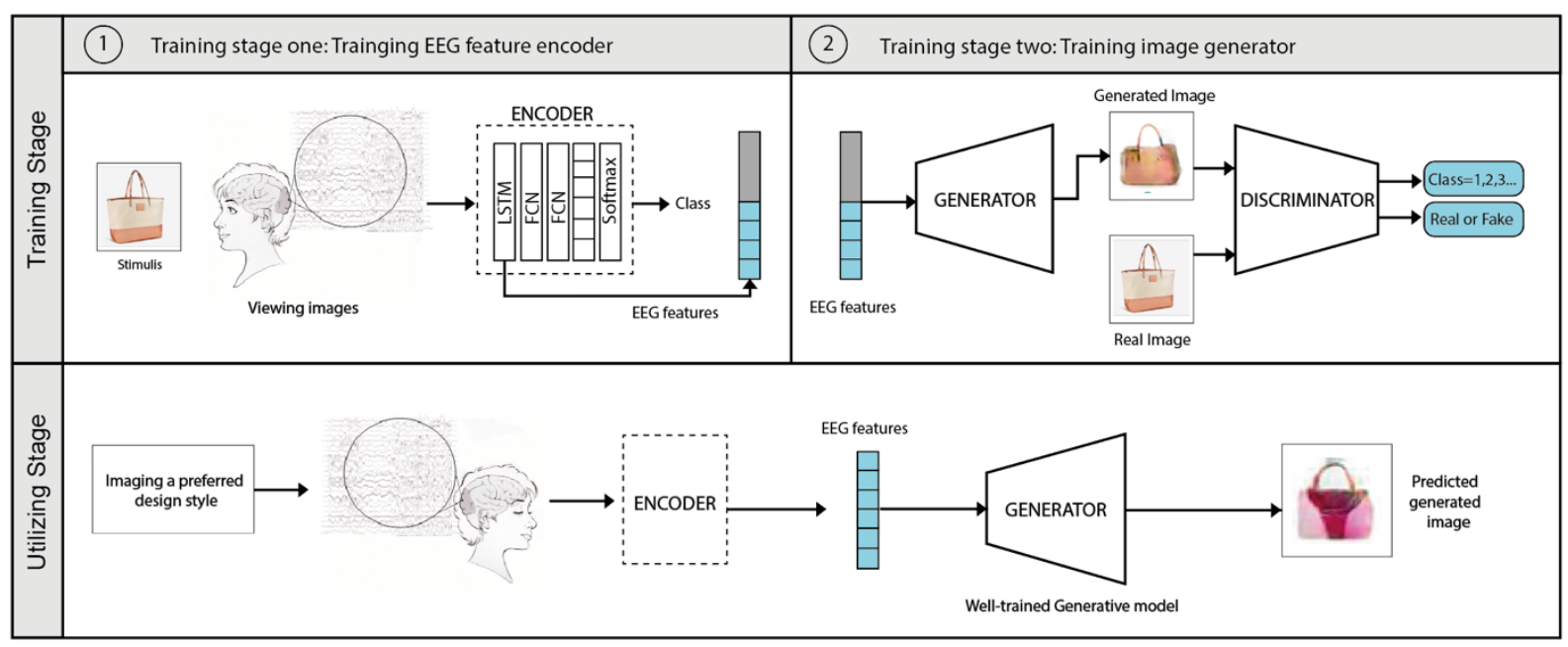

Figure 1. Overview of the process of brain signal conditioned design image generation.

\section{RELATED WORK}

Three scientific areas have inspired this research. In this section state-of-the-art machine learning technology for generative creativity and problems with the current machine generated design methods are described. Secondly current neuroscience inspired design methods are explored. The third area considered concerns using deep neural networks to classify, generate and reconstruct visual images from brain activities (EEG \& fMRI). Taking inspiration from these three areas of study a framework is proposed where brain activities are adopted as input to introduce human cognition in a GAN based generative design process.

\subsection{Deep learning for design}

Several deep neural network approaches for image generation have been proposed recently, such as the neural style transfer model (Gatys et al., 2016) (Johnson et al., 2016; Li \& Wand 2016) (Zhu et al., 2017) which can generate images which contain the content of the given image with style features from the artistic images. Isola et al (Isola et al., 2017b) investigate the image-to-image problem which generates new images from photos applied to human-drawn sketches. These approaches have enabled the development of computer vision applications, for example, Prisma (Anon n.d.), a photo editor which turns a photo to an artwork. Dong et al. have synthesised realistic image semantics with natural language descriptions (Dong et al., 2017). Yu et al. (Yu et al., 2018) use an approach called DesignGAN to generate a shape-oriented bionic design which maintains the shape of the design target and combines the features from the biological source domain. These approaches mainly focus on automatically generating new art and design images with the features from input images. A problem of this type of generative creativity is evaluation since the generation is completely random. How to generate a desirable design with the preference from clients is a key question in our research.

\subsection{Current neuroscience-inspired design}

Non-invasive methods for measuring human brain activity include Electroencephalograph (EEG), functional magnetic resonance imaging (fMRI) and Near-Infrared Spectroscopy (NIRS). EEG measures subcranial electrical signals from electrodes in contact with the scalp. Neuroscience has inspired many developments in design, such as understanding cognitive neurofeedback from clients, building and developing new products and evaluating advertising. For example, neuroimaging has been used in understanding of packaging design to explain how packaging design confuses the consumer (Basso et al., 2014). Velasco et al. have presented an experimental research programme on evaluating the impact of different orientation of design elements in product packaging (Velasco et al., 2015). Furthermore, to understand the consumer psychology of a brand, Plassmann et al. have reviewed the applications of marketing and also describe issues for future research (Plassmann et al., 2012). In a review of neuroscience-inspired design (Spence 2016) states one of the problems of commercial neuromarketing is that the results provided by neuroimaging are a clear answer to a 'black-and-white' question rather than a discriminating analysis of a 'shades of grey' question. 
Inspired by his review, the potential has been explored of introducing neuroscience into a deep learning framework where the machine could not only provide a response to a 'black-and-white' question but also show other potential visualisations relating to a 'shade of grey' intuition.

\subsection{Brain signal conditioned deep learning framework}

Machine learning methods have been introduced to both EEG and fMRI to help understand visual images, for example, developing a visual object classifier driven by human brain signal (Spampinato et al., 2017). Distinct from Spampinato et al. who used EEG data, Horikawa et al. explore object decoding using machine learning principles, predicted from fMRI patterns (Horikawa \& Kamitani 2017). Both of these EEG and fMRI results show the potential of brain-based information retrieval. Furthermore, researchers have tried to generate related visual information from decoded information from brain imaging. Palazzo et al. have combined generative adversarial networks with a recurrent neural network model to process EEG signals to generate viewing images of participants (Palazzo et al., 2017). Recently, Shen et al. have successfully demonstrated that visual images can be reconstructed from decoded fMRI signals (Shen et al., 2017; Shen et al., 2018). These generative brain decoding methods provide inspiration to explore a new method for design cognitive analysis.

\section{METHOD: HUMAN-IN-THE-LOOP DESIGN WITH MACHINE LEARNING FRAMEWORK}

How to involve human cognition into a random AI design process to generate a design with people's preference is the research focus presented here. Human cognition is captured by measuring EEG signals. The process includes two phases: a training phase to learn a generating function $G_{B D}: B \rightarrow D$ which maps the EEG measured brain activity $B$ to the corresponding design image $D$, and a design phase to utilise the learned generating function and particular brain signal to generate a product involving the human preference.

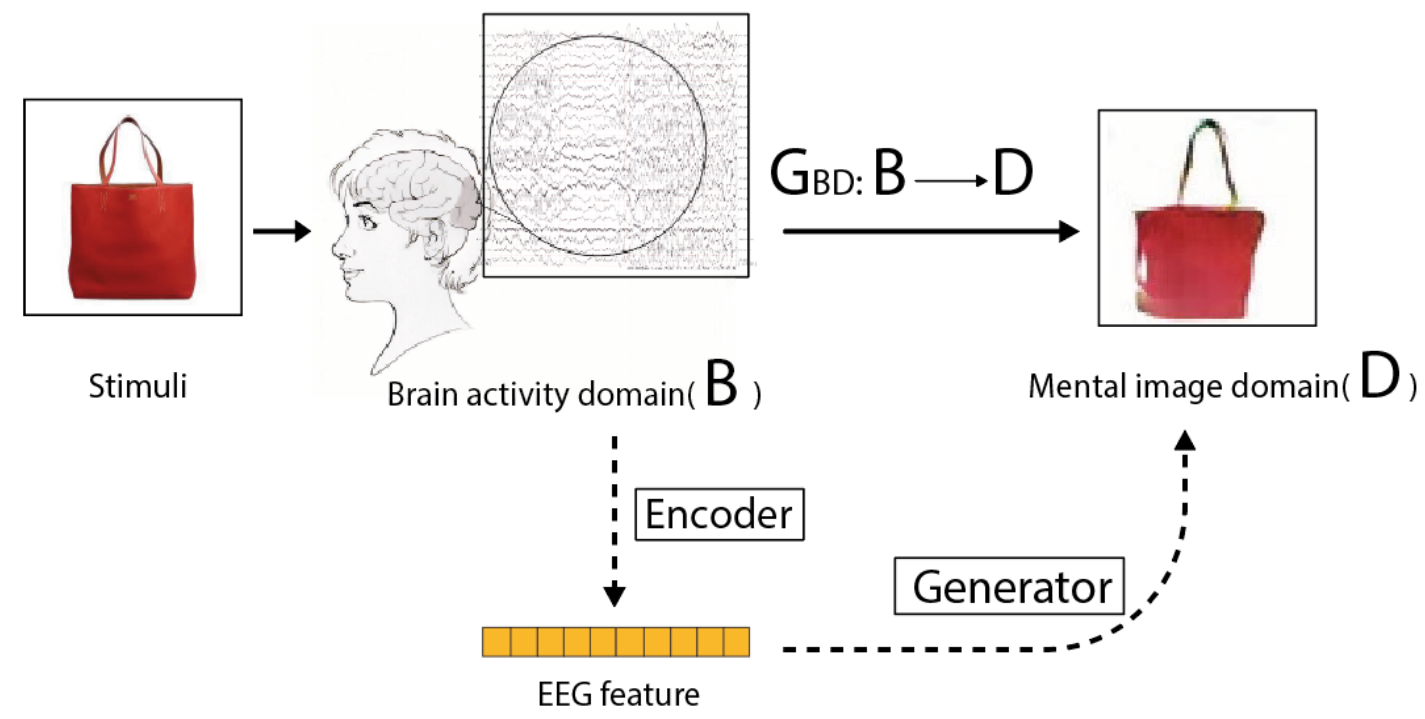

Figure 2. Training an EEG conditioned generative model.

In the training stage, EEG signals were recorded when subjects were viewing the 'ground-truth' images of a design. Subsequently the brain signals $B_{i}$ are encoded into the EEG features related to the design semantic of the seen image by a LSTM-based EEG encoder. The EEG features are embedded into the GAN-based generator as the generation condition, which forces the generative model to reconstruct images $D_{i}$ that contain the same design semantic of the original seen image. In the design stage, the subjects are asked to imagine an example of a product or a design they prefer, and the measured EEG signal which may contain favoured design features of the subject's will then be encoded as the input to the trained generator. The sampled design containing the design features that correspond to the subject's imagination will then be created by the generator. Figure 2 illustrates how the EEG encoder and image generator can be trained. Details about how this framework is implemented will be introduced in the following sections. 


\section{EXPERIMENT IMPLEMENTATION}

Details of the experiments for the model training process are presented in this section.

\subsection{Participants and equipment}

The EEG study included 6 right-handed student volunteers ( 3 females and 3 males) aged between 1730 years old, with normal or corrected-to-normal vision. All participants gave informed consent to take part in the EEG experiment and had considerable training in EEG experiments. Our EEG recordings were performed using an electrode cap with $64 \mathrm{Ag} / \mathrm{AgCI}$ electrodes which were mounted according to the extended international 10/20 system. An online $50 \mathrm{~Hz}$ notch filter was added to avoid power line signal contamination.

Signals were recorded by using a Neuroscan Synamp2 Amplifier (Scan 4.3.1; Neurosoft Labs Inc., Sterling, Virginia, USA) and sampled at $1000 \mathrm{~Hz}$. Eye blinks were recorded from left supra-orbital and infra-orbital electrodes, whereas the horizontal eye movement EEG was recorded from electrodes placed $15 \mathrm{~mm}$ laterally to the left and right external canthi. The forehead (AFZ) was used for the ground electrode, and the reference electrode was attached to the left mastoid. All electrode's impedances were maintained below $5 \mathrm{k} \Omega$.

\subsection{Visual stimuli}

In this experiment, the stimuli consisted of 5 different categories of product images (handbag, headset, mug, watch and guitar) from ImageNet (Fei-Fei et al., 2010); each category included 50 images. The size of the pictures was resized to 500x500 pixels and cropped to the centre of the screen. The participants were required to view more images for brain signal decoding to capture more features from visual images.

\subsection{Experiment design}

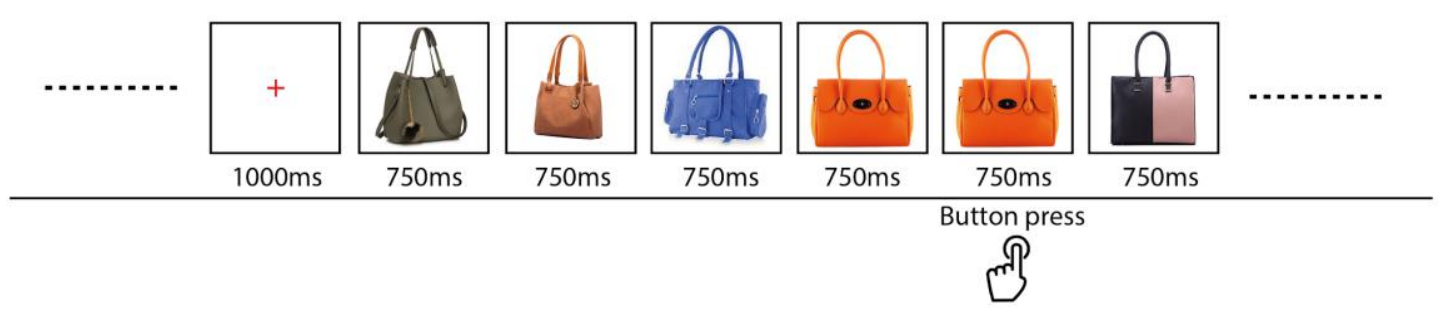

Figure 3. Image presentation experiment.

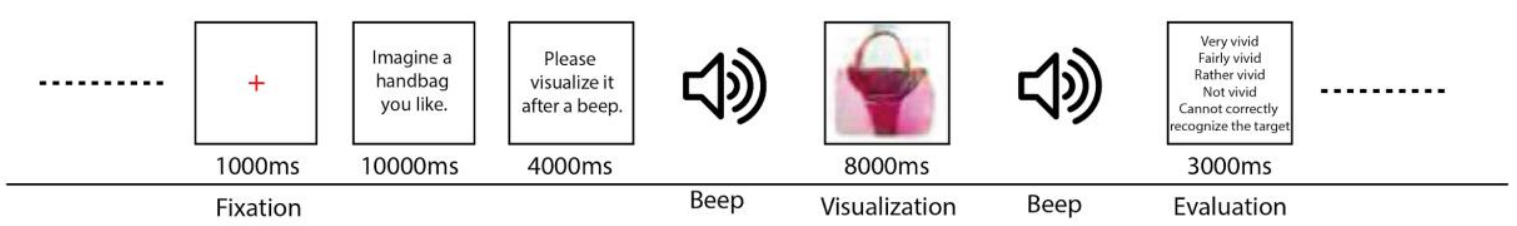

Figure 4. Preference imagery experiment.

Two separate data collection sessions were conducted consisting of an image presentation experiment and the preference imagery session. In order to ensure the quality of the data, an electrode connection checking session was added before each run. During the experiment the subject was accommodated in a sound attenuated and electrically shielded room and seated comfortably. The stimuli images were presented in the centre on the screen and at a fixed distance. In addition, a press button pad was provided for the subjects to give feedback during the experiment. Subjects were able to stop the experiment at any time.

In the image presentation session, 5 categories of images were presented in 5 runs, each run consisting of 1 category of 50 images and separated in 5 blocks, each block with 10 different images and 1 repeated image. The subjects were required to view the images and press the button on the board when 
they sew the repeated images to maintain their attention. At the beginning of each block, a fixation red cross was presented in the central of the screen for $1000 \mathrm{~ms}$. At the end of each run, $3000 \mathrm{~ms}$ were added as a rest time. In the preference imagery experiment, the subjects were required to visually imagine their preferred products follow the instructions appears on the screen. This session consisted of 5 runs and each run contained 10 blocks. Firstly, a fixation red cross was shown in the centre of the screen for $1000 \mathrm{~ms}$. After this, an instruction was presented in the middle of the screen, and the subjects were asked to visualize the preferred visual look of the product. Following an audible beep, they were asked to close their eyes for an $8 \mathrm{~s}$ imagination period. After this, the subjects were required to evaluate the correctness and vividness of their mental imagery on a five-point scale by pressing the button of the box. $3000 \mathrm{~ms}$ refreshing time was added before and after each run.

\subsection{Generative model}

\subsubsection{EEG feature encoder}

The objective of this work is to map the stimulated brain signals into the correct latent representation of seen images, and thus to build a model to extract EEG features as correlated to the image features as possible.

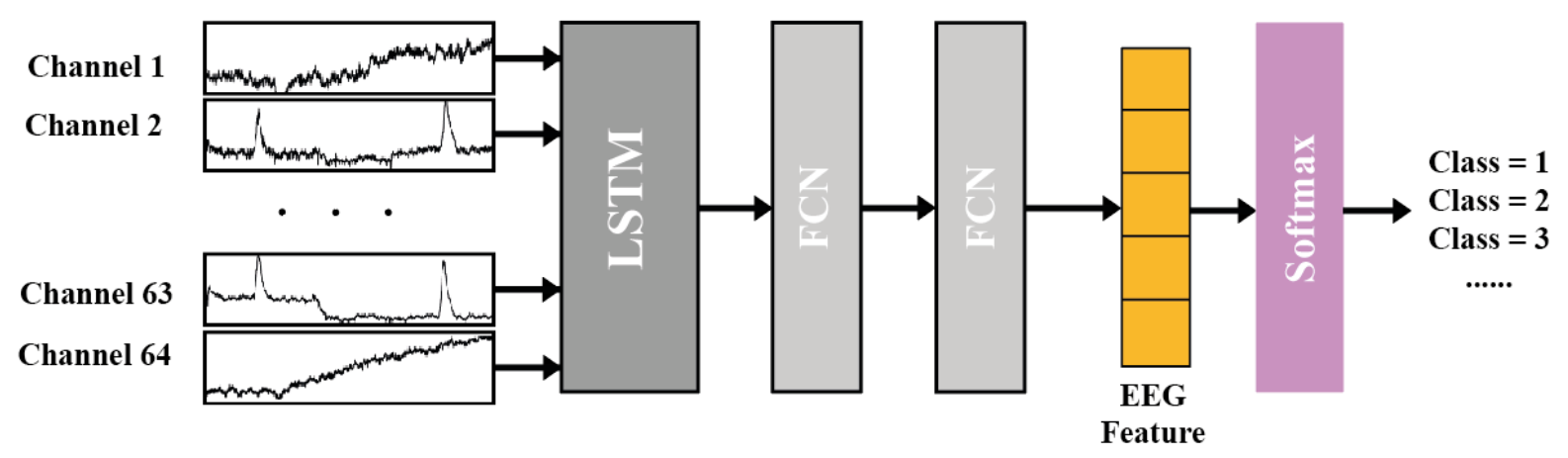

Figure 5. EEG feature encoder.

A recurrent neural network using Long Short-Term Memory (LSTM) (Hochreiter and Schmidhuber 1997) cell was employed to track the temporal dynamics in the EEG data which contains fundamental information for EEG activity comprehension. LSTM is a special recurrent neural network which is able to learn long-term dependencies. Figure 5 illustrates the architecture of our EEG feature encoder. This is made up of a standard LSTM layer and two fully-connected layers (linear combinations of input, followed by ReLU nonlinearity). At each time step $t$, the data of all EEG channels at time $t$ is fed into the LSTM layer; The output of the LSTM layer at the last time step is used as the input of the fully-connected layers, ReLU nonlinearity is appended after the first fully-connected layer and a Softmax layer is appended after the last fully-connected layer. The learning rate is initialized to 0.0001 and gradient descent is used to learn the model's parameters end-to-end. The dataset is split into 3 sets: 80\% EEG data for training, 10\% EEG data for validation, $10 \%$ EEG data for testing. Table 1 illustrates the classification rate among five classes, with a total of 1500 EEG data points (300 per class), which includes 1200 data points for training, 150 data points for validation and 150 data points for testing. The overall classification rate on the test set which contains 5 classes is $71.5 \%$.

Table 1. Classification rate for each class.

\begin{tabular}{|c|c|}
\hline Class name & Classification rate \\
\hline Headphone & $84.2 \%$ \\
\hline Bag & $79.2 \%$ \\
\hline Mug & $70.8 \%$ \\
\hline Guitar & $67.5 \%$ \\
\hline Watch & $55.8 \%$ \\
\hline Total & $71.5 \%$ \\
\hline
\end{tabular}




\subsubsection{Generator network}

The foundation of the generator framework is ACGAN (Odena et al., 2016) . This generates images based on the input feature vector and also has the ability to generate images from the specific category.

\section{Generator}

The generator consists of 5 upsampling layers. Firstly, inputs of the EEG representation which is the element-wise product of the 64-dimensional EEG features and a random Gaussian noise have been made. The input vector is then spatially upsampled by four times by the first transposed convolutional layers and output 512 feature maps. After that, the number of feature map halves and the feature map size doubles after each remaining transposed convolutional layer. Finally, the final output has been obtained as the $64 \times 64$ pixel images with three colour channels. Batch normalisation (Ioffe and Szegedy 2015) and LeakyReLU (Maas et al., 2013) nonlinearities have been appended after each transposed convolutional layer.

\section{Discriminator}

The discriminator consists of two modules: a convolutional module used to extract the image feature and a classification module used to distinguish generated image and identify the image category as well.

Convolutional module. The convolutional part of the discriminator is made up of 10 convolutional layers. It takes as input coloured 64 x 64 images. We have 64 feature maps after the first layer and the number of feature maps reaches 512 after being doubled at layer 3,5 and 8 respectively. The feature map size starts at $64 \times 64$ and is halved after each max pooling layer appended after the 2, 4, 7, 10 layers and become $4 \times 4$ after the final layer. Batch normalisation and LeakyReLU nonlinearities are appended after each convolutional layer.

Classification module. After the convolutional module, a $4 \times 4 \times 512$ sized data sample is obtained. The data is flattened and fed into two classifiers, a binary classifier to distinguish generated images from the real image and a multi-class classifier to identify the image category. The binary classifier consists of two fully connected layers. After the first layer, the output size is 1024 and 1 after the second layer. ReLU activation function is appended after the first fully-connected layer, and a sigmoid layer is added after the second fully-connected layer. The multi-class classifier consists of three fullyconnected layers. The first layer reduces the number of features to 1024 and the features number remain unchanged after the second layer. Then, the data is fed into the last layer where the number of features is reduced to the number of image categories. ReLU activation function is appended after the first and the second layer and a Softmax layer is added after the last fully-connected layer.

The Inception score (Salimans et al., 2016) is applied as the main method to evaluate the quality of the images generated by the GAN. A score of 4.85 was obtained on the generated image in this study.

\subsection{Evaluation experiment}

A questionnaire survey was conducted in order to provide some evaluation of the method. In this human study survey, subjects were required to choose images they like from 100 images ( 5 classes, 20 images per class) which were randomly chosen from the generated results. 6 subjects who had participated in the brain signal recording experiments evaluated these images. For each trial of the experiment, the participants viewed a printing of a set of generated images and were required to pick the images they like. 


\section{RESULTS AND ANALYSIS}

\subsection{Results}

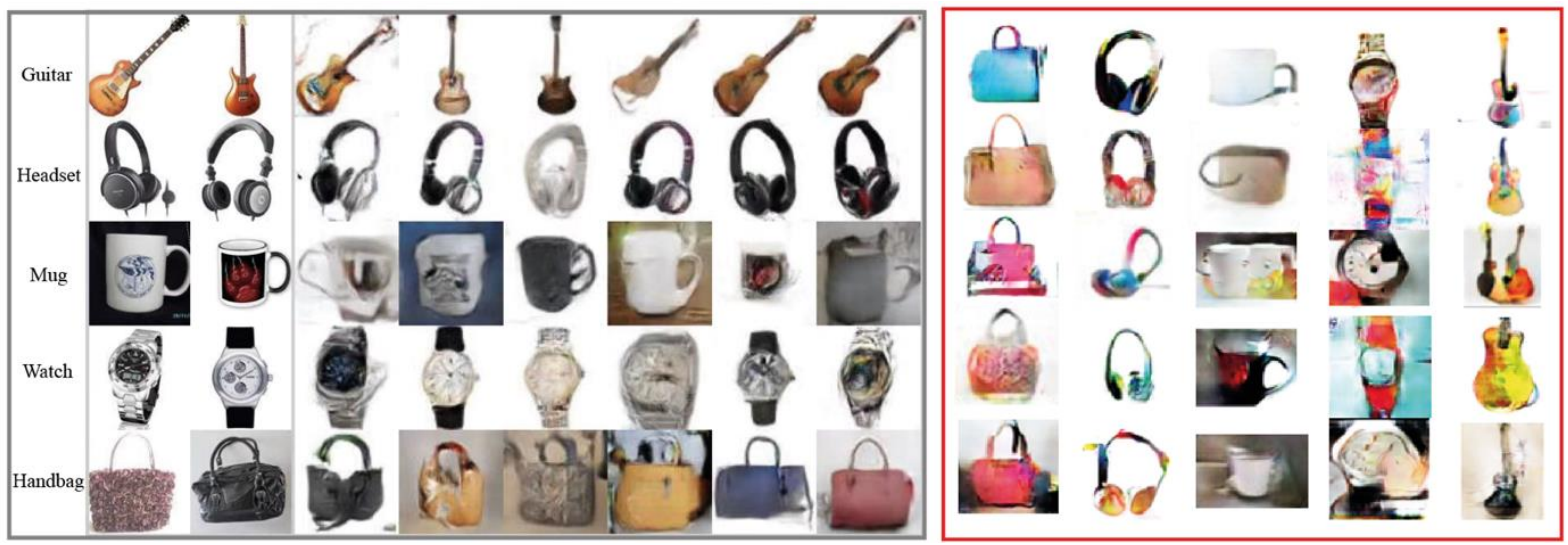

Figure 6. Seen image reconstruction results in the grey frame (left) and imagery preference design image in red frame (right) reconstruction results.

Results from both the image presentation experiment and the preference imagery experiment are shown in Figure 6. The as seen image results from the image presentation experiment are shown in the grey frame in Figure 6, as a baseline of the work to allow for subsequent evaluation of the performance of the visual image reconstruction model. After reconstructing the seen image from the image presentation experiment, the well-trained model is used to reconstruct the imagery image from the preference imagery experiment, which is shown in the red frame in Figure 6.

\section{Qualitative and quantitative evaluations}

To evaluate the brain signal conditioned generative design results, both qualitative and quantitative evaluations were performed. In the qualitative evaluation, it can be seen from the generated results demonstrated that the proposed approach successfully generates different designs with multiple colour and shape features. As mentioned in section 4.4.1, the overall classification rate of the encoder is 78.5\% and in section 4.4.2 the inception score of the generator is 4.85. The quantitative evaluation result is from the questionnaire survey. Figure 7 shows there are $73 \%$ bag images, $60 \%$ headphone images, $61 \%$ mug images, $51 \%$ watch images and $43 \%$ guitar images that have been selected by the participants as the designs they like. In total, there were $57.6 \%$ images chosen from the generated image as preferred images.

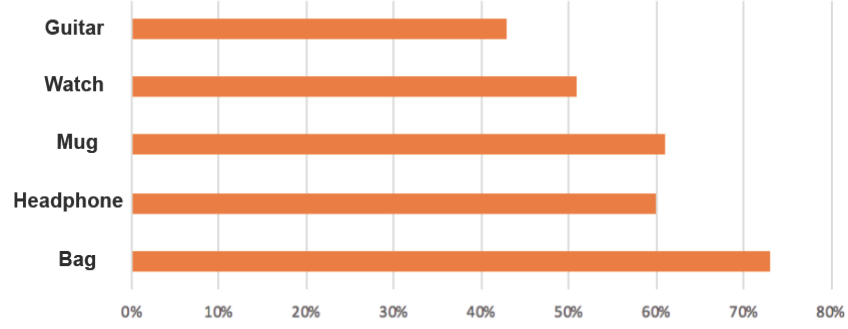

Figure 7. Human evaluation results of the case study.

The evaluation results indicate that these generated design images which are conditioned by brain activities could include human preferred design styles. The reconstructed imagined images have a larger variety of colour and shape features than the reconstructed seen image. The preference imagery experiment results also shows that these preferred products generated by deep learning method through brain activities have combined multiple design features from various kinds of products which learned from previous designs. Therefore, it may be inferred that these generated designs contain the mixed colour and shape features which have been filtered by human cognition by inputting brain signal into a deep generative model. Designers could have a prejudgment based on these generated images. For example, one of the generated bags has multiple colours, from which we could predict 
that the user actually wants a very lively bag. Similarly with the grey bag, we could predict an office style bag is what they might prefer. Such a discriminating analysis of a 'shades of grey' design question could be applied to different design processes. Product designs dominated by the shape are more accepted than the designs dominated by function such as a guitar. This may reflect that the preference of shape is better captured by EEG signal. Further study of this hypothesis could provide additional evidence and insights into this finding.

\section{CONCLUSION}

In this paper, a Human-in-the-loop design method has been proposed with machine learning to automatically generate a design taking into account a person's preference. The case study results have indicated that designs can be generated with preferred design styles by using the image reconstruction model conditioned by mental imagery EEG features. Although the proposed approach has only been applied for five product design cases, it could potentially be used in other design cases and for different design tasks such as design evaluation and branding strategy. In the research work to date, due to the limited data in the model training process, the case study only contains the design semantics from these five categories. Data in additional categories can be collected in order to contain more features. The results may help designers think beyond user cases by having direct visualistion of what the user may like. The experiment indicates a new way of communicating human cognitive content. Embedding the proposed Human-in-the-loop design method into different design processes could help designers understand users' requirements and preferences more accurately. A new approach to design cognition analysis has been demonstrated.

\section{REFERENCES}

Anon, Prisma, Available at: https://prisma-ai.com/ [Accessed November 5, 2018].

Basso, F. et al. (2014), "Why people drink shampoo? Food Imitating Products are fooling brains and endangering consumers for marketing purposes". PloS one, Vol. 9 No. 9, p. e100368.

Carroll, J.M. (2002), "Scenarios and design cognition". In Proceedings IEEE Joint International Conference on Requirements Engineering. ieeexplore.ieee.org, pp. 3-5.

Cooley, M. (2000), "Human-centered design". Information design, pp. 59-81.

Dong, H. et al. (2017), "Semantic Image Synthesis via Adversarial Learning", In 2017 IEEE International Conference on Computer Vision (ICCV). Available at: http://dx.doi.org/10.1109/iccv.2017.608.

Dosovitskiy, A. and Brox, T. (2016), "Generating Images with Perceptual Similarity Metrics based on Deep Networks", arXiv [cs.LG]. Available at: http://arxiv.org/abs/1602.02644.

Efros, A.A. and Freeman, W.T. (2001), "Image quilting for texture synthesis and transfer", In Proceedings of the 28th annual conference on Computer graphics and interactive techniques - SIGGRAPH '01. Available at: http://dx.doi.org/10.1145/383259.383296.

Fei-Fei, L., Deng, J. and Li, K. (2010), "ImageNet: Constructing a large-scale image database", Journal of vision, Vol. 9 No. 8, pp. 1037-1037.

Gatys, L.A., Ecker, A.S. and Bethge, M. (2016), "Image Style Transfer Using Convolutional Neural Networks", In 2016 IEEE Conference on Computer Vision and Pattern Recognition (CVPR). Available at: http://dx.doi.org/10.1109/cvpr.2016.265.

Goodfellow, I. et al. (2014),“Generative Adversarial Nets”, In Z. Ghahramani et al., eds. Advances in Neural Information Processing Systems Vol. 27. Curran Associates, Inc., pp. 2672-2680.

Horikawa, T. and Kamitani, Y. (2017), "Generic decoding of seen and imagined objects using hierarchical visual features", Nature communications, 8, p. 15037.

Ioffe, S. and Szegedy, C. (2015), "Batch Normalization: Accelerating Deep Network Training by Reducing Internal Covariate Shift", arXiv [cs.LG]. Available at: http://arxiv.org/abs/1502.03167.

Isola, P. et al. (2017a), "Image-to-image translation with conditional adversarial networks", arXiv preprint. Available at: http://openaccess.thecvf.com/content_cvpr_2017/papers/Isola_Image-ToImage_Translation_With_CVPR_2017_paper.pdf.

Isola, P. et al. (2017b), "Image-to-Image Translation with Conditional Adversarial Networks", In 2017 IEEE Conference on Computer Vision and Pattern Recognition (CVPR). Available at: http://dx.doi.org/10.1109/cvpr.2017.632.

Johnson, J., Alahi, A. and Fei-Fei, L. (2016), "Perceptual Losses for Real-Time Style Transfer and SuperResolution", In Lecture Notes in Computer Science. pp. 694-711.

Li, C. and Wand, M., (2016), "Precomputed Real-Time Texture Synthesis with Markovian Generative Adversarial Networks", In Lecture Notes in Computer Science. pp. 702-716. 
Maas, A.L., Hannun, A.Y. and Ng, A.Y. (2013), "Rectifier nonlinearities improve neural network acoustic models", In in ICML Workshop on Deep Learning for Audio, Speech and Language Processing. Available at: http://citeseerx.ist.psu.edu/viewdoc/summary?doi=10.1.1.693.1422 [Accessed December 14, 2018].

Odena, A., Olah, C. and Shlens, J. (2016), "Conditional Image Synthesis With Auxiliary Classifier GANs", arXiv [stat.ML]. Available at: http://arxiv.org/abs/1610.09585.

Palazzo, S. et al. (2017), “Generative Adversarial Networks Conditioned by Brain Signals”. In 2017 IEEE International Conference on Computer Vision (ICCV). Available at: http://dx.doi.org/10.1109/iccv.2017.369.

Plassmann, H., Ramsøy, T.Z. and Milosavljevic, M. (2012), "Branding the brain: A critical review and outlook", Journal of consumer psychology: the official journal of the Society for Consumer Psychology, Vol. 22 No. 1 , pp. 18-36.

Salimans, T. et al. (2016), "Improved techniques for training gans", In Advances in Neural Information Processing Systems. pp. 2234-2242.

Shen, G. et al. (2017), Deep Image Reconstruction from Human Brain Activity. Available at: http://dx.doi.org/10.1101/240317.

Shen, G. et al. (2018), End-To-End Deep Image Reconstruction from Human Brain Activity. Available at: http://dx.doi.org/10.1101/272518.

Spampinato, C. et al. (2017), “Deep Learning Human Mind for Automated Visual Classification”, In Proceedings of the IEEE Conference on Computer Vision and Pattern Recognition. pp. 6809-6817.

Spence, C. (2016), "Neuroscience-Inspired Design: From Academic Neuromarketing to Commercially Relevant Research", Organizational Research Methods, pp. 1-24.

Tirupattur, P. et al. (2018), "ThoughtViz: Visualizing Human Thoughts Using Generative Adversarial Network" In 2018 ACM Multimedia Conference on Multimedia Conference. ACM, pp. 950-958.

Velasco, C., Woods, A.T. and Spence, C. (2015), "Evaluating the orientation of design elements in product packaging using an online orientation task", Food quality and preference, Vol. 46, pp. 151-159.

Vicente, K.J. (2013), The Human Factor: Revolutionizing The Way People Live with Technology, Routledge.

Yu, S. et al. (2018), "Generative Creativity: Adversarial Learning for Bionic Design", arXiv [cs. CV]. Available at: http://arxiv.org/abs/1805.07615.

Zhu, J.-Y. et al. (2017), "Unpaired Image-to-Image Translation Using Cycle-Consistent Adversarial Networks", In 2017 IEEE International Conference on Computer Vision (ICCV). Available at: http://dx.doi.org/10.1109/iccv.2017.244.

\section{ACKNOWLEDGMENTS}

The authors would like to acknowledge Zhejiang University Neuromanagement Lab and ALP project who helped to collect EEG data. 